\title{
HARSH TRUTH: DO HEALTHCARE PROVIDERS SILENCE WOMEN WHO EXPERIENCE INTIMATE PARTNER VIOLENCE
}

\author{
Bijaya Pokharel, Kathy Hegadoren, Elisavet \\ Papathanassoglou
}

Faculty of Nursing, University of Alberta, Edmonton, Canada

\begin{abstract}
Background: Globally, one-third of women experience abuse from their intimate partners. Since intimate partner violence (IPV) creates a chronic stress environment, affected women suffer from several physical and mental stress-related disorders for which they seek healthcare services in higher proportion to that of non-abused women. Although affected women seek help for the consequences of IPV, addressing the cause, is an important responsibility of healthcare providers. This study aimed to explore how healthcare providers may contribute to silencing of women who have experienced IPV.

Subjects and Methods: This was an integrative review. We performed a systematic search of eight databases for articles published between 2007 and 2018. We identified 4507 publications. We included the English language articles that focused on adult women between 18 and 49 years of age, explored the issue of silencing of women who have experienced IPV, and followed a primary research study design. Two reviewers screened the articles using the web application, Rayyan. Quality was assessed using Joanna Briggs Institute Critical Appraisal tools.

Results: Five articles were selected for analysis. The findings revealed that healthcare providers might play a significant role in silencing women's suffering from abuse. Affected women's unwillingness to act as their own agent in healthcare settings or disclose experiences of IPV was associated with healthcare provider's inadequate or inappropriate response. Lack of affirmation, validation, and inability to make women feel accepted were the main factors which silenced women who experienced IPV. Both individual-level factors, such as shame, fear, humiliation, hope, and relationship dynamics, and community-level factors, such as cultural norms, and values, seemed to precede the factors related to healthcare providers.

Conclusion:A socio-ecological understanding of the factors influencing silencing of women who have experienced IPV is required. A health care model which takes into consideration the contributing factors at various ecological levels of influence is imperative to guide healthcare providers towards the development of best practices in caring with women who have experienced violence in their intimate relationships.
\end{abstract}

Keywords: intimate, partner, violence, health care, provider

\section{Correspondence:}

Bijaya Pokharel. Faculty of Nursing, University of Alberta, Edmonton, Canada.

Email: bijaya@ualberta.ca.Mobile: +17803991038. 